\title{
THEORIES AND ANTECEDENTS OF KNOWLEDGE SHARING BEHAVIOUR IN VIRTUAL COMMUNITY: A SYSTEMATIC REVIEW
}

\author{
Muhammad Ashraf Fauzi \\ Faculty of Industrial Management, \\ Universiti Malaysia Pahang, Malaysia \\ Nor Rokiah Hanum Md Haron \\ Faculty of Industrial Management, Universiti Malaysia Pahang, Malaysia \\ Fatin Izzati Ramli \\ Faculty of Industrial Management, Universiti Malaysia Pahang, Malaysia \\ Hamirahanim Abdul Rahman \\ Faculty of Industrial Management, Universiti Malaysia Pahang, Malaysia \\ Zetty Ain Kamaruzzaman \\ Faculty of Industrial Management, Universiti Malaysia Pahang, Malaysia
}

\begin{abstract}
Virtual Community (VC) is regarded as the best platform for professionals in various fields to share their expertise and knowledge. Since the escalation of web 2.0 and the internet within the last decade and the booming interest in big data and expansion of industry 4.0, VC is deemed as an ideal proxy for practitioners to share and earned instant knowledge that can beimplemented within business activities and day to day application. Despite this emerging interest, there has been no comprehensive study on the overall antecedents of KS in VC. Applying a systematic review, a total of 68 relevant articles that discusses knowledge sharing (KS) via VC are evaluated. Several central themes of theories applied in this field within the literature are discussed on its importance and relevance. Important antecedents arealso reviewed on its practicality and implementation in understanding the role of KS in VC. The implication of this review would benefit stakeholders in maintaining the sustainability of VC as the platform for a knowledge-based society.
\end{abstract}

Keywords: Theories, Antecedents, Knowledge Management, Knowledge Sharing, Virtual Community.

\section{INTRODUCTION}

Knowledge is vital in ensuring that organizationsand industries to sustain in the current challenging business world. As the world shifted towards the advancement of internet and digitalization, virtual platform has served as a vibrant tool in knowledge distribution. Virtual communities (VC) provide a platform for users to communicate and interact without having to meet face to face (Chang et al., 2015). Practically, employees have been found to seek knowledge by consulting their peers through various virtual networks and social media rather than accessing knowledge database and repository (Akhavan et al. 2015; Djelantik, 2019). Hence, the proposition and availability of VC have provided employees the chance to interact and look out for peers that can give instant knowledge by the community through the web.

Many of the previous studies have focused on the aspect of individual, organizational, and technological factors (Al-Kurdi et al., 2018). The three elements are embedded within the underpinning theories applied in KS studies throughout the literature. With the extensive studies on VC as the emerging area of knowledge management, there have been various theories implemented in understanding the KS behavior among members in this community (Fauzi et al., 2018). Among the commonly used theories are related to social-related theories such as social capital, social cognitive, social exchange, and social identity. Other theories that are known to the knowledge management communities 
are the theory of planned behaviour and theory of reasoned action. In understanding the underlying foundation of member's engagement on KS within $\mathrm{VC}$, relevant and related theories should be recognized and correlated among each other'sin developing a holistic model consisting of relevant antecedents of KS in VC.

With this in mind, this paper tends to review all related studies from the year 2006-2019, thirteen years on KS within the VC. The main objective of this review is to provide insight and an in-depth understanding of the theories and essential antecedents that have been applied in the literature associated with the user's KS behaviour in VC. In parallel with today's digital development, knowledge should be shared freely and safely to ensure that implicit and explicit knowledge could be captured for the benefits of members in VC. Hence, a knowledge-based society can be enhanced and maintained for the betterment of society.

Knowledge sharing in virtual community $\mathrm{KS}$ is a process where knowledge, expertise, experience, skills, and information that is exchanged between families, friends, communities, and organizations (Charband and Navimipour 2016). Virtual or online knowledge communities consist of people having the same interest, profession, or goals. They share and interchange knowledge for their use in daily life by engaging in social interaction (Feng and Ye, 2016). People use VC not only for knowledge exchange but also a tool for retrieving information, social support, entertainment, and also recreation (Phang et al., 2009; Fauzi, 2019).

Despite that VC is widely research and applied, the term and notion of VC application differ from one industry and context to another (Bolisani and Scarso, 2014).Several scholars have termed it as community of practice (Zboralski et al, 2009, Jeon et al, 2011a; Hau et al 2013; Chu et al., 2014; Tseng \& Kuo, 2014; Nistor et al., 2015), virtual community of practice (Usoro et al, 2007; Fang \& Chu, 2010; Majewski et al., 2011, Chang et al., 2016), professional virtual community (Lin et al., 2009; Chen \& Hung, 2010; Chiu et al., 2011; Tamjidyamcholo et al., 2014), online communities (Ma \& Agarwal, 2007; Phang et 1., 2009; Chai \& Kim, 2010, Erden et al, 2012;
Lai \& Chen, 2014), online knowledge communities (Ye et al., 2015; Feng \& Ye, 2016), online question and answer community (Jin et al., 2013; Khansa et al 2015; Guan et al., 2018), online health community (Yan et al., 2016; Zheng et al., 2017), virtual innovation community (Zhang et al., 2017a; Pirkkalainen et al., 2018), online user community (Hau \& Kang, 2016), online discussion communities (Kumi \& Sabherwal, 2018), online travel community (Ku, 2012; Yuan et al., 2016), peer to peer problem solving (P3) virtual community (Zhao et al 2013) and virtual learning community (Chen et al., 2009; Lu et al., 2013). As there are many terminologies used within this area, VC will be used throughout this paper as the majority of studies had used this term as it is more general and involveda broad definition describing the aspect of a virtual platform.

The theoretical framework of this review is based on the frequently adapted theories used in VC. These include the theory of planned behavior/reasoned action, technology acceptance model, social capital theory, social cognitive theory, social identity theory, social exchange theory, expectancy disconfirmation theory, motivation theory, and use \& gratification theory. All the studies included apply at least one theory or integration of two or more, that could facilitate in understanding $\mathrm{KS}$ behavior in VC.

\section{METHODOLOGY}

This section delineates the method in assessing this systematic review of KS within the scope of VC. The review includes all articles within the indexed journal of Scopus and Web of science. The eight databases that were included in the review include Science Direct, Emerald Insight, Taylor \& Francis, Wiley Blackwell, Springer-Link, IEEE Explore, Wiley \& Blackwell, Inderscience, and JSTOR. These databases are selected because they are reliable database and has an extensive record of peer-reviewed articles within the scope of VC. The process in retrieving articles related to Knowledge sharing in VC. The author used the PRISMA method (Moher et al., 2015). This method enables the ease of the systematic review by having exclusion criteria, review process steps, data analysis, and abstraction.PRISMA serves as a checklist to clearly explain the need for a specific topic (Wormald \& Evans, 2018). It adds to the future 
avenues prioritizing on the fundamental aspect of VC knowledge sharing context.

PRISMA

\section{Identification}

The first process is keyword identification. Searching process for related and identical terminologies. These terms and synonym were searched through dictionaries, thesaurus and encyclopaedia. The search string used in this study includes the keyword ("knowledge sharing" OR "knowledge exchange" OR "knowledge transfer" OR "knowledge distribution" OR "knowledge acquisition") AND ("virtual community" OR "online community" OR "community of practice" OR "professional virtual community" OR "virtual team"). The initial result had found a total of 1,502 articles having keywords, as stated. After screening using PRISMA methodology, the final relevant studies accepted for review were 68 . Table 1 presents the study selection process results.

Table 1: Study selection process results.

\begin{tabular}{|l|c|c|}
\hline Online database & $\begin{array}{c}\text { Initial } \\
\text { result }\end{array}$ & $\begin{array}{c}\text { Relevant } \\
\text { studies }\end{array}$ \\
\hline Science Direct & 243 & 30 \\
\hline Emerald Insight & 127 & 8 \\
\hline Springer-Link & 234 & 4 \\
\hline Taylor \& Francis & 486 & 12 \\
\hline IEEE Explore & 117 & 2 \\
\hline Wiley \& Blackwell & 237 & 6 \\
\hline Inderscience & 49 & 1 \\
\hline JSTOR & 9 & 2 \\
\hline $\begin{array}{l}\text { Google Scholar/ other } \\
\text { publishers (second } \\
\text { stage screening) }\end{array}$ & - & 3 \\
\hline Total & 1,502 & 68 \\
\hline
\end{tabular}

Screening

Before the articles are accepted for review, the inclusion and exclusion criteria process was conducted to screen the articles. Based on article type, only articles with empirical data are taken. Review article, book chapter, conference proceeding is not included. Secondly, only articles in English are accepted, to avoid problems in translating and having originally written articles in English. Thirdly, all the studies are from the period of 13 years, from 2006 to 2019. This is considered to be adequate time in viewing research evolution in the VC field of study. Lastly, studies that are chosen within the context of VC or related terminology are accepted. Studies from other disciplines and contexts are excluded. Table 2 summarizes the criteria for this review.

Table 2: Inclusion and exclusion criteria

\begin{tabular}{|c|c|}
\hline $\begin{array}{c}\text { Inclusion criteria } \\
\text { article }\end{array}$ & Exclusion criteria \\
\hline Written in English & Other than English \\
\hline $\begin{array}{c}\text { Within the domain of } \\
\text { virtual community } \\
\text { and other related } \\
\text { terminology }\end{array}$ & Qualitative study \\
\hline $\begin{array}{c}\text { From the year } 2006 \text { to } \\
2019\end{array}$ & \\
\hline
\end{tabular}

\section{Eligibility}

A total of 69 articles made up to this stage. This stage requires the authors to screen related article based on the title and abstract. It is a second stage screening to ensure that the articles included are based on KS in VC studies. A total of 68 articles were included for analysis.Figure 1 summarizes the four-stage process of the PRISMA method, indicating identification, screening, eligibility, and finally, included articles.

\section{RESULT}

\section{Overview of studies}

All the articles were searched in 8 databases, resulting in 68 studies. The summary of the reviews is shown in table 2. Most of the studies were found in computer cyberpsychology journals, with other areas including management and social psychology. The majority were conducted in the Asia Pacific region that includes Taiwan, South Korea, Hong Kong, and China. Other prominent countries have the USA, Singapore, Malaysia, Australia, and several European countries. Respondents of the studies vary from professional, students, teachers, teenagers, and the common public using VC as education and entertainment purpose. Most of the studies applied related social theories by integrating 2 or 3 theories with the minority using only one theory or underpinning framework. Table 3 summarizes the 68 articles found from this review. 


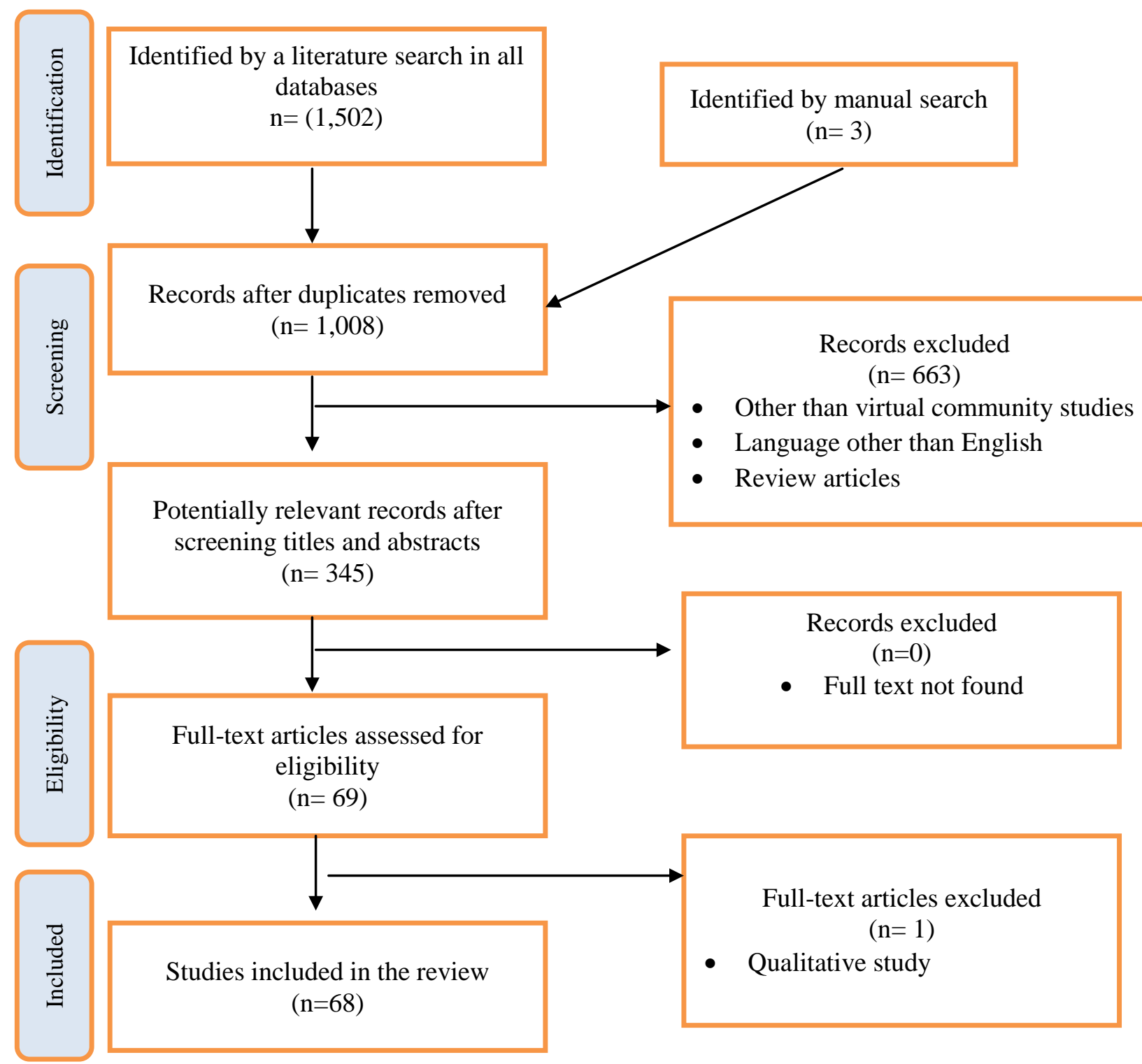

Figure 1: PRISMA diagram Four-phased flow diagram of studies' selection procedure

Table 3: Summary of knowledge sharing in virtual community studies

\begin{tabular}{|c|c|c|c|c|c|c|}
\hline No & Authors & Sample & $\begin{array}{l}\text { Country/ } \\
\text { countries }\end{array}$ & Term used & $\begin{array}{c}\text { Theory/ theories/ } \\
\text { framework }\end{array}$ & Antecedents \\
\hline 1. & $\begin{array}{l}\text { Chiu et al. } \\
\text { (2006) }\end{array}$ & $\begin{array}{l}310 \text { members from } \\
\text { one professional } \\
\text { virtual community }\end{array}$ & Taiwan & $\begin{array}{c}\text { Virtual } \\
\text { communities }\end{array}$ & $\begin{array}{c}\text { social capital } \\
+ \\
\text { social-cognitive }\end{array}$ & \begin{tabular}{lr}
\multicolumn{3}{l}{ Social interaction ties, trust, } \\
norm of reciprocity, \\
identification, & shared \\
language, shared & vision, \\
personal & outcome \\
expectations, community- & remater \\
related & outcome \\
expectations & \\
\end{tabular} \\
\hline 2. & $\begin{array}{l}\text { Ma \& Agarwal } \\
\text { (2007) }\end{array}$ & $\begin{array}{l}666 \text { from two } \\
\text { online } \\
\text { communities }\end{array}$ & $\begin{array}{l}\text { USA } \\
\end{array}$ & $\begin{array}{c}\text { Online } \\
\text { communities }\end{array}$ & $\begin{array}{c}\text { attribution theory } \\
+ \\
\text { self-presentation } \\
\text { theory }\end{array}$ & $\begin{array}{l}\text { Virtual co-presence, } \\
\text { persistent labeling, self- } \\
\text { presentation, deep profiling, } \\
\text { tenure, offline activity, } \\
\text { satisfaction, information } \\
\text { need fulfillment, group } \\
\text { identification, offline } \\
\text { activity. }\end{array}$ \\
\hline 3. & $\begin{array}{l}\text { Usoro et al. } \\
\text { (2007) }\end{array}$ & $\begin{array}{l}75 \quad \text { community } \\
\text { members }\end{array}$ & $\begin{array}{c}\text { US (45\%), } \\
\text { UK (34\%) } \\
\text { and Australia } \\
(11 \%) . \\
\text { Switzerland, }\end{array}$ & $\begin{array}{c}\text { virtual } \\
\text { communities of } \\
\text { practice }\end{array}$ & Trust factors & $\begin{array}{l}\text { Three categories of trust: } \\
\text { Integrity-basedtrust } \\
\text { Competence-basedtrust } \\
\text { Benevolence-basedtrust }\end{array}$ \\
\hline
\end{tabular}




\begin{tabular}{|c|c|c|c|c|c|c|}
\hline No & Authors & Sample & $\begin{array}{l}\text { Country/ } \\
\text { countries }\end{array}$ & Term used & $\begin{array}{l}\text { Theory/ theories/ } \\
\text { framework }\end{array}$ & Antecedents \\
\hline & & & $\begin{array}{c}\text { Spain, } \\
\text { Denmark, and } \\
\text { India }(10 \%) .\end{array}$ & & & \\
\hline 4. & Hsu et al. (2007) & 274 respondents & $\begin{array}{c}\text { Taiwan, } \\
\text { Hong Kong, } \\
\text { and China }\end{array}$ & $\begin{array}{l}\text { Virtual } \\
\text { community }\end{array}$ & $\begin{array}{l}\text { Social cognitive } \\
\text { theory }\end{array}$ & $\begin{array}{lr}\text { Trust } & \text { (economy, } \\
\text { information-based, } \\
\text { identification) } \\
\text { expectation-efficacy, } \\
\text { community) }\end{array}$ \\
\hline 5. & $\begin{array}{l}\text { Staples \& } \\
\text { Webster (2008) }\end{array}$ & 824 respondents & Canada & Virtual team & $\begin{array}{l}\text { Social exchange } \\
\text { theory }\end{array}$ & $\begin{array}{l}\text { Task interdependence, trust, } \\
\text { virtualness, } \\
\text { effectiveness }\end{array}$ \\
\hline 6. & Lin et al. (2009) & $\begin{array}{l}\text { Three } r \text { hundred } \\
\text { fifty respondents } \\
\text { from } 3 \text { club } \\
\text { (Programmer- } \\
\text { Club, Blue- } \\
\text { Shop, and Pure C) }\end{array}$ & Taiwan & $\begin{array}{l}\text { Professional } \\
\text { virtual } \\
\text { communities } \\
\text { (PVCs) }\end{array}$ & $\begin{array}{l}\text { Social cognitive } \\
\text { theory }\end{array}$ & $\begin{array}{lr}\text { Trust, } & \text { self-efficacy, } \\
\text { perceived } & \text { relative } \\
\text { advantage, and } & \text { perceived } \\
\text { compatibility. } & \end{array}$ \\
\hline 7. & $\begin{array}{l}\text { Phang et al. } \\
\text { (2009) }\end{array}$ & $\begin{array}{l}115 \text { students from } \\
\text { computing course }\end{array}$ & China & $\begin{array}{c}\text { Online } \\
\text { communities }\end{array}$ & $\begin{array}{l}\text { Value theory } \\
+ \\
\text { Social exchange } \\
\text { theory }\end{array}$ & $\begin{array}{l}\text { Ease of use, system } \\
\text { reliability, knowledge } \\
\text { tracking fulfillment, social } \\
\text { interactivity, perception of } \\
\text { the moderator, perceived } \\
\text { usability, perceived } \\
\text { sociability }\end{array}$ \\
\hline 8. & Zboralski 2009 & $\begin{array}{l}222 \text { CoP members } \\
\text { multinational } \\
\text { company }\end{array}$ & Germany & $\begin{array}{l}\text { Community of } \\
\text { practice (CoPs) }\end{array}$ & $\begin{array}{l}\text { interaction } \\
\text { frequency } \\
\text { (notheory) }\end{array}$ & $\begin{array}{lr}\text { Members' } & \text { motivation, } \\
\text { community } & \text { leader, } \\
\text { management } & \text { support, } \\
\text { interaction } & \text { frequency, } \\
\text { interaction quality }\end{array}$ \\
\hline 9. & $\begin{array}{l}\text { Chen et al. } \\
(2009)\end{array}$ & $\begin{array}{l}396 \text { MBA students } \\
\text { enrolled in virtual } \\
\text { courses. }\end{array}$ & Taiwan & $\begin{array}{l}\text { Virtual learning } \\
\text { communities }\end{array}$ & $\begin{array}{c}\text { Theory of planned } \\
\text { behavior } \\
+ \\
\text { SCT (social } \\
\text { network ties) } \\
\end{array}$ & $\begin{array}{l}\text { Knowledge creation and } \\
\text { web-specific self-efficacy, } \\
\text { social network ties, TPB } \\
\text { variable }\end{array}$ \\
\hline 10. & $\begin{array}{l}\text { Fang and Chiu } \\
(2010)\end{array}$ & $\begin{array}{ll}142 & \text { IT-oriented } \\
\text { VCoP } & \end{array}$ & Taiwan & $\begin{array}{c}\text { Virtual } \\
\text { community of } \\
\text { practice (VCoP) }\end{array}$ & $\begin{array}{c}\text { Social exchange } \\
\text { theory } \\
+ \\
\text { organizational } \\
\text { citizenship } \\
\text { behaviors (OCB)- }\end{array}$ & $\begin{array}{l}\text { Justice, trust, altruism, } \\
\text { personality } \\
\text { (conscientiousness) }\end{array}$ \\
\hline 11. & $\begin{array}{lll}\text { Zhang } & \text { et } & \text { al. } \\
(2010) & & \end{array}$ & 144 professionals & China & $\begin{array}{c}\text { Virtual } \\
\text { communities }\end{array}$ & $\begin{array}{l}\text { Psychological } \\
\text { safety }\end{array}$ & $\begin{array}{l}\text { Psychological safety, Trust } \\
\text { Self-consciousness }\end{array}$ \\
\hline 12. & Yu et al. (2010) & $\begin{array}{l}442 \text { from } 3 \text { online } \\
\text { communities }\end{array}$ & Taiwan & $\begin{array}{c}\text { Virtual } \\
\text { community }\end{array}$ & $\begin{array}{l}\text { three community } \\
\text { sharing cultural } \\
\text { factors: fairness, } \\
\text { identification } \\
\text { and openness }\end{array}$ & $\begin{array}{l}\text { fairness, identification, and } \\
\text { openness, enjoy helping, } \\
\text { usefulness/relevancy }\end{array}$ \\
\hline 13. & Cho et al. (2010) & 223 respondents & Singapore & $\begin{array}{c}\text { Wikipedia } \\
\text { community/virtual } \\
\text { community }\end{array}$ & $\begin{array}{c}\text { Theory of planned } \\
\text { behavior } \\
+ \\
\text { motivations theory } \\
+ \\
\text { cognitive belief } \\
+ \\
\text { social-relational } \\
\text { factors }\end{array}$ & 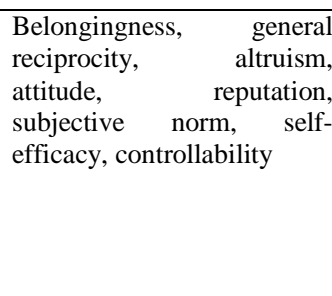 \\
\hline 14. & $\begin{array}{l}\text { Chai and Kim } \\
\text { (2010) }\end{array}$ & $\begin{array}{l}485 \text { respondents } \\
\text { (bloggers) }\end{array}$ & $\begin{array}{l}\text { United States } \\
\text { of America }\end{array}$ & $\begin{array}{c}\text { Online } \\
\text { community }\end{array}$ & $\begin{array}{l}\text { trust in multiple } \\
\text { dimensions }\end{array}$ & $\begin{array}{l}\text { Trust: categorized into } \\
\text { bloggers, economy, trust in } \\
\text { the Internet, trust in a blog } \\
\text { service provider }\end{array}$ \\
\hline 15. & $\begin{array}{l}\text { Chen and Hung } \\
2010\end{array}$ & $\begin{array}{l}323 \text { members of } \\
\text { two } \\
\text { communities }\end{array}$ & Taiwan & $\begin{array}{l}\text { Professional } \\
\text { virtual } \\
\text { communities } \\
\text { (PVCs) }\end{array}$ & $\begin{array}{l}\text { Social cognitive } \\
\text { theory }\end{array}$ & $\begin{array}{l}\text { Norm of reciprocity, } \\
\text { interpersonal trust, self- } \\
\text { efficacy, perceived related } \\
\begin{array}{l}\text { advantage, } \\
\text { compatibility. }\end{array}\end{array}$ \\
\hline 16. & $\begin{array}{l}\text { Shen et al. } \\
\text { (2010) }\end{array}$ & $\begin{array}{l}430 \text { registered } \\
\text { Members from } 4 \\
\text { VCs }\end{array}$ & $\begin{array}{l}\text { United Arab } \\
\text { Emirates }\end{array}$ & $\begin{array}{c}\text { Virtual } \\
\text { communities }\end{array}$ & $\begin{array}{c}\text { social presence } \\
\text { theory } \\
+ \\
\text { social identity } \\
\text { theory } \\
\end{array}$ & $\begin{array}{l}\text { Awareness, affective, social } \\
\text { presence, cognitive, social } \\
\text { presence }\end{array}$ \\
\hline
\end{tabular}




\begin{tabular}{|c|c|c|c|c|c|c|}
\hline No & Authors & Sample & $\begin{array}{l}\text { Country/ } \\
\text { countries }\end{array}$ & Term used & $\begin{array}{c}\text { Theory/ theories/ } \\
\text { framework }\end{array}$ & Antecedents \\
\hline 17. & $\begin{array}{l}\text { Chiu et al. } \\
\text { (2011) }\end{array}$ & $\begin{array}{l}270 \text { members of a } \\
\text { professional } \\
\text { virtual community }\end{array}$ & Taiwan & $\begin{array}{c}\text { Professional } \\
\text { virtual community }\end{array}$ & $\begin{array}{c}\text { Expectancy } \\
\text { disconfirmation } \\
\text { theory } \\
+ \\
\text { social network } \\
\text { factors } \\
+ \\
\text { justice theory }\end{array}$ & $\begin{array}{l}\text { Playfulness, satisfaction, } \\
\text { disconfirmation, justice }\end{array}$ \\
\hline 18. & Hsu et al. (2011) & $\begin{array}{l}324 \text { technical VC } \\
\text { members }\end{array}$ & Taiwan & $\begin{array}{c}\text { Virtual } \\
\text { communities }\end{array}$ & $\begin{array}{l}\text { Trust building } \\
\text { factors }\end{array}$ & $\begin{array}{l}\text { Trust (in members, in the } \\
\text { system), knowledge growth, } \\
\text { perceived responsiveness, } \\
\text { social interaction ties, } \\
\text { shared vision, system } \\
\text { quality, and knowledge } \\
\text { quality }\end{array}$ \\
\hline 19. & $\begin{array}{l}\text { Kim } \\
(2011)\end{array}$ et al. & $\begin{array}{l}185 \text { Members of } \\
\text { Cyworld (a virtual } \\
\text { community of } \\
\text { relationships, } \\
\text { interests, and } \\
\text { transactions) }\end{array}$ & $\begin{array}{l}\text { South Korea } \\
\text { and a few } \\
\text { other } \\
\text { countries } \\
\text { using } \\
\text { Cyworld }\end{array}$ & $\begin{array}{l}\text { Virtual } \\
\text { community- } \\
\text { blogging } \\
\text { communities }\end{array}$ & $\begin{array}{l}\text { Social identity } \\
\text { theory }\end{array}$ & $\begin{array}{l}\text { Involvement, kindness, } \\
\text { social skills, creativity }\end{array}$ \\
\hline 20. & $\begin{array}{l}\text { Jeon et al. } \\
(2011 \mathrm{a})\end{array}$ & $\begin{array}{l}\text { formal } \\
\text { members } \\
\text { and } \\
\text { informal } \quad \text { CoP } \\
\text { members }(157) \\
\mathrm{N}=282\end{array}$ & South Korea & $\begin{array}{l}\text { Community of } \\
\text { practice (CoPs) }\end{array}$ & $\begin{array}{c}\text { Theory of Planned } \\
\text { Behaviour } \\
+ \\
\text { Motivation Theory } \\
+ \\
\text { Triandis model }\end{array}$ & $\begin{array}{l}\text { Extrinsic motivation (image, } \\
\text { reciprocity), intrinsic } \\
\text { motivation (enjoyment in } \\
\text { helping, need for } \\
\text { affiliation), type of CoP, } \\
\text { attitude, PBC, subjective } \\
\text { norm }\end{array}$ \\
\hline 21. & $\begin{array}{l}\text { Majewski et al. } \\
2011\end{array}$ & 152 respondents & $\begin{array}{l}\text { USA, UK, } \\
\text { Netherlands, } \\
\text { Germany, } \\
\text { Canada, and } \\
\text { Australia }\end{array}$ & $\begin{array}{l}\text { Virtual } \\
\text { community of } \\
\text { practice }\end{array}$ & $\begin{array}{l}\text { Social factors } \\
\text { (trust, norm of } \\
\text { reciprocity) }\end{array}$ & $\begin{array}{l}\text { Norms of reciprocity, trust, } \\
\text { perception of community, } \\
\text { knowledge provision and } \\
\text { reception }\end{array}$ \\
\hline 22. & $\begin{array}{l}\text { Shu \& Chuang. } \\
(2011)\end{array}$ & $\begin{array}{l}217 \text { respondents } \\
\text { online virtual } \\
\text { communities }\end{array}$ & Taiwan & $\begin{array}{c}\text { Virtual } \\
\text { communities }\end{array}$ & $\begin{array}{l}\text { Theory of reasoned } \\
\text { action }\end{array}$ & $\begin{array}{l}\text { Expected return, absorption } \\
\text { capacity, organization-based } \\
\text { self-esteem, trust, attitude }\end{array}$ \\
\hline 23. & $\begin{array}{l}\text { Jeon et al. } \\
(2011 \mathrm{~b}) \text {. }\end{array}$ & $\begin{array}{l}179 \text { members from } \\
70 \text { CoPs of a large } \\
\text { multinational } \\
\text { electronics firm }\end{array}$ & South Korea & $\begin{array}{l}\text { Communities of } \\
\text { practice }\end{array}$ & $\begin{array}{c}\text { Triandis model } \\
+ \\
\text { expectancy } \\
\text { theory of } \\
\text { motivation } \\
\text { (perceived } \\
\text { consequences) }\end{array}$ & \begin{tabular}{lr} 
Facilitating & \multicolumn{2}{c}{ conditions, } \\
social factors, affect \\
anticipated recognition, \\
anticipated & reciprocal \\
relationship, & anticipated \\
usefulness, & perceived \\
consequence. &
\end{tabular} \\
\hline 24. & $\begin{array}{l}\text { Yoon and } \\
\text { Rolland (2012) }\end{array}$ & 209 VC users & South Korea & $\begin{array}{c}\text { Virtual } \\
\text { communities }\end{array}$ & $\begin{array}{c}\text { Self-determination } \\
\text { theory }\end{array}$ & $\begin{array}{lr}\text { Familiarity, anonymity, } \\
\text { perceived competence, } \\
\text { perceived autonomy, } \\
\text { perceived relatedness }\end{array}$ \\
\hline 25. & Xu et al. (2012) & $\begin{array}{l}199 \text { Chinese } \\
\text { undergraduates } \\
\text { and } 200 \text { USA } \\
\text { undergraduates }\end{array}$ & $\begin{array}{l}\text { China and the } \\
\text { United States } \\
\text { of America }\end{array}$ & $\begin{array}{c}\text { Virtual } \\
\text { communities }\end{array}$ & $\begin{array}{l}\text { Social cognitive } \\
\text { theory }\end{array}$ & $\begin{array}{lr}\text { Attachment } & \text { motivation, } \\
\text { trust, social } & \text { support } \\
\text { orientation } & \end{array}$ \\
\hline 26. & $\begin{array}{l}\text { Erden et al. } \\
(2012)\end{array}$ & $\begin{array}{l}531 \text { Online photo } \\
\text { community }\end{array}$ & South Korea & $\begin{array}{l}\text { Online } \\
\text { community }\end{array}$ & $\begin{array}{l}\text { Theory of planned } \\
\text { behavior }\end{array}$ & $\begin{array}{l}\text { Community munificence, } \\
\text { attitude, subjective norm, } \\
\text { perceived } \\
\text { control. }\end{array}$ \\
\hline 27. & Ku 2012 & $\begin{array}{l}235 \text { online travel } \\
\text { communities }\end{array}$ & Taiwan & $\begin{array}{l}\text { Online travel } \\
\text { community }\end{array}$ & $\begin{array}{c}\text { Social identity } \\
\text { theory } \\
+ \\
\text { Technology } \\
\text { acceptance model }\end{array}$ & $\begin{array}{l}\text { Trust, commitment, website } \\
\text { design quality, website } \\
\text { service quality }\end{array}$ \\
\hline 28. & Hau et al. (2013) & $\begin{array}{l}2010 \text { respondents } \\
\text { from multiple } \\
\text { industries }\end{array}$ & South Korea & $\begin{array}{l}\text { Community of } \\
\text { practice }\end{array}$ & $\begin{array}{c}\text { Rational action } \\
\text { theory } \\
+ \\
\text { Social capital }\end{array}$ & $\begin{array}{l}\text { Organizational } \begin{array}{l}\text { reward, } \\
\text { reciprocity, } \\
\text { social capital } \\
\text { (social tie, }\end{array} \\
\text { social trust, social goals }\end{array}$ \\
\hline
\end{tabular}




\begin{tabular}{|c|c|c|c|c|c|c|}
\hline No & Authors & Sample & $\begin{array}{l}\text { Country/ } \\
\text { countries }\end{array}$ & Term used & $\begin{array}{c}\text { Theory/ theories/ } \\
\text { framework }\end{array}$ & Antecedents \\
\hline & & & & & theory & \\
\hline 29. & Chen et al., 2013 & $\begin{array}{l}219 \text { professional } \\
\text { IT VC }\end{array}$ & Taiwan & $\begin{array}{c}\text { peer-to-peer } \\
\text { problem solving } \\
\text { (P3) Virtual } \\
\text { Community } \\
\end{array}$ & $\begin{array}{c}\text { uses and } \\
\text { gratification theory } \\
\text { (U\&G theory) }\end{array}$ & $\begin{array}{l}\text { Entertainment. Social need, } \\
\text { information need, attitude }\end{array}$ \\
\hline 30. & $\begin{array}{l}\text { Liao et al. } \\
(2013)\end{array}$ & $\begin{array}{l}473 \text { undergraduate } \\
\text { and } \\
\text { graduatesstudents }\end{array}$ & Taiwan & $\begin{array}{c}\text { virtual } \\
\text { communities }\end{array}$ & $\begin{array}{l}\text { Social exchange } \\
\text { theory }\end{array}$ & $\begin{array}{l}\text { Self-efficacy, reward, } \\
\text { reciprocity, reputation, } \\
\text { enjoying helping, expected } \\
\text { relationship, sharing culture } \\
\text { (fairness, identification, } \\
\text { openness), attitude }\end{array}$ \\
\hline 31. & $\begin{array}{l}\text { Lin and Huang } \\
(2013)\end{array}$ & 167 respondents & Taiwan & $\begin{array}{c}\text { Virtual } \\
\text { communities }\end{array}$ & $\begin{array}{l}\text { Theory of reasoned } \\
\text { action }\end{array}$ & $\begin{array}{l}\text { self-efficacy, altruism, } \\
\text { reward, and the sense of } \\
\text { virtual community }\end{array}$ \\
\hline 32. & Yan et al. (2013) & $\begin{array}{l}232 \text { users of Web } \\
2.0 \quad \text { virtual } \\
\text { communities }\end{array}$ & China & $\begin{array}{c}\text { Virtual } \\
\text { communities }\end{array}$ & $\begin{array}{l}\text { Self-perception } \\
\text { theory }\end{array}$ & $\begin{array}{l}\text { Perceived enjoyment, } \\
\text { attention focus, employee } \\
\text { creativity }\end{array}$ \\
\hline 33. & $\begin{array}{l}\text { Hung \& Cheng } \\
\text { (2013) }\end{array}$ & $\begin{array}{l}218 \text { members from } \\
\text { My3q website }\end{array}$ & Taiwan & $\begin{array}{c}\text { Virtual } \\
\text { communities }\end{array}$ & $\begin{array}{c}\text { Technology } \\
\text { acceptance model } \\
+ \\
\text { Innovation } \\
\text { Diffusion Theory }\end{array}$ & 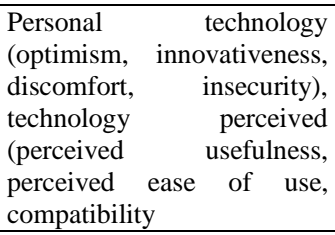 \\
\hline 34. & Zhao et al. 2013 & $\begin{array}{l}185 \text { patients from } \\
3 \text { diseased focused } \\
\text { POC }\end{array}$ & $\begin{array}{l}\text { United States } \\
\text { of America }\end{array}$ & $\begin{array}{l}\text { Patient online } \\
\text { communities } \\
\text { (POC) }\end{array}$ & $\begin{array}{l}\text { Social identity } \\
\text { theory }\end{array}$ & $\begin{array}{l}\text { Trust, social identity, } \\
\text { empathy }\end{array}$ \\
\hline 35. & $\begin{array}{l}\text { Shan et al. } \\
\text { (2013) }\end{array}$ & $\begin{array}{lr}205 \text { teachers } & \text { and } \\
\text { students } & \text { from } \\
\text { colleges } & \text { and } \\
\text { universities } & \end{array}$ & China & $\begin{array}{l}\text { Virtual } \\
\text { community }\end{array}$ & $\begin{array}{c}\text { social cognitive } \\
\text { theory } \\
+ \\
\text { social capital } \\
\text { theory } \\
+ \\
\text { characteristics of } \\
\text { emergency events }\end{array}$ & $\begin{array}{l}\text { Emergency events, shared } \\
\text { vision, shared language, } \\
\text { social interaction ties, } \\
\text { outcome expectations, } \\
\text { identification, trust. }\end{array}$ \\
\hline 36. & $\begin{array}{l}\text { Papadopoulos et } \\
\text { al. (2013) }\end{array}$ & $\begin{array}{lr}175 & \text { respondents } \\
\text { from } & \text { Thailand } \\
\text { weblogs } & \end{array}$ & Thailand & $\begin{array}{l}\text { Virtual } \\
\text { communities } \\
\text { (weblogs) }\end{array}$ & $\begin{array}{c}\text { social influence } \\
\text { theory } \\
+ \\
\text { technology } \\
\text { acceptance } \\
+ \\
\text { social cognitive } \\
\text { theory } \\
+ \\
\text { individual factors }\end{array}$ & $\begin{array}{l}\text { Subjective norm, social } \\
\text { identity, group norm, } \\
\text { perceived } \\
\text { perceived ease of use, } \\
\text { perceived enjoyment, self- } \\
\text { efficacy, personal outcome } \\
\text { expectation, altruism, } \\
\text { attitude }\end{array}$ \\
\hline 37. & Lu et al. 2013 & $\begin{array}{l}321 \text { academics of a } \\
\text { training program }\end{array}$ & China & $\begin{array}{l}\text { Virtual learning } \\
\text { community } \\
\text { (VLCs) }\end{array}$ & $\begin{array}{l}\text { Social cognitive } \\
\text { theory }\end{array}$ & $\begin{array}{l}\text { Instructors, peers, } \\
\text { management, self-efficacy, } \\
\text { outcome expectance }\end{array}$ \\
\hline 38. & Jin et al. 2013 & $\begin{array}{lr}241 & \text { respondents } \\
\text { from } & \text { Yahoo! } \\
\text { Answer } & \text { China } \\
\text { community } & \end{array}$ & China & $\begin{array}{l}\text { Online question } \\
\text { answering } \\
\text { communities }\end{array}$ & $\begin{array}{l}\text { Expectation } \\
\text { confirmation } \\
\text { theory }\end{array}$ & $\begin{array}{l}\text { Reputation enhancement, } \\
\text { reciprocity, enjoyment in } \\
\text { helping others, knowledge } \\
\text { self-efficacy, confirmation, } \\
\text { satisfaction, continuance } \\
\text { intention }\end{array}$ \\
\hline 39. & Chu et al. 2014 & $\begin{array}{l}120 \text { respondents } \\
\text { from a company }\end{array}$ & Australia & $\begin{array}{l}\text { Communities of } \\
\text { practice (CoPs) }\end{array}$ & Personality traits & Personality traits \\
\hline 40. & $\begin{array}{l}\text { Tamjidyamcholo } \\
\text { et al. (2014) }\end{array}$ & $\begin{array}{lr}142 & \text { respondents } \\
\text { online r from } \\
\text { LinkedIn }\end{array}$ & Malaysia & $\begin{array}{l}\text { professional } \\
\text { virtual } \\
\text { communities } \\
\text { (PVC) }\end{array}$ & $\begin{array}{c}\text { Triandis model } \\
+ \\
\text { expectancy } \\
\text { theory of } \\
\text { motivation } \\
\text { (perceived } \\
\end{array}$ & $\begin{array}{l}\text { perceived consequences, } \\
\text { affect, social factor, } \\
\text { facilitating conditions }\end{array}$ \\
\hline
\end{tabular}




\begin{tabular}{|c|c|c|c|c|c|c|}
\hline No & Authors & Sample & $\begin{array}{l}\text { Country/ } \\
\text { countries }\end{array}$ & Term used & $\begin{array}{c}\begin{array}{c}\text { Theory/ theories/ } \\
\text { framework }\end{array} \\
\end{array}$ & Antecedents \\
\hline & & & & & consequences) & \\
\hline 41. & Zeng et al. 2014 & $\begin{array}{l}211 \text { members of } \\
\text { the online hotel } \\
\text { industry }\end{array}$ & China & $\begin{array}{c}\text { Virtual } \\
\text { communities }\end{array}$ & $\begin{array}{c}\text { Social cognitive } \\
\text { theory } \\
+ \\
\text { social capital } \\
\text { theory } \\
+ \\
\text { Social identity } \\
\text { theory }\end{array}$ & $\begin{array}{l}\text { Social interaction ties, trust, } \\
\text { norm of reciprocity, } \\
\text { identification, shared vision, } \\
\text { expected results, self- } \\
\text { efficacy, loyalty }\end{array}$ \\
\hline 42. & $\begin{array}{l}\text { Tseng \& Kuo } \\
(2014)\end{array}$ & $\begin{array}{l}321 \text { teachers from } \\
\text { largest CoPs in } \\
\text { Taiwan }\end{array}$ & Taiwan & $\begin{array}{l}\text { Community of } \\
\text { practice (CoPs) }\end{array}$ & $\begin{array}{c}\text { Social Capital } \\
\text { Theory } \\
+ \\
\text { Social Cognitive } \\
\text { Theory }\end{array}$ & $\begin{array}{l}\text { Tie strength, self-efficacy, } \\
\text { altruistic commitment, } \\
\text { performance expectation }\end{array}$ \\
\hline 43. & $\begin{array}{l}\text { Lai \& Chen } \\
(2014)\end{array}$ & $\begin{array}{l}324 \quad(n=146 \text { for } \\
\text { poster and } n=178 \\
\text { for lurker })\end{array}$ & Taiwan & $\begin{array}{c}\text { Online } \\
\text { communities }\end{array}$ & Value theory & $\begin{array}{lr}\text { Reputation, } & \text { reciprocity, } \\
\text { enjoyment in helping, self- } \\
\text { efficacy, } \\
\text { moderator's } & \text { perceived } \\
\text { offline } & \text { enthusiasm, } \\
\text { enjoyability. } & \text { activities, }\end{array}$ \\
\hline 44. & $\begin{array}{l}\text { Tsai \& Bagozzi } \\
(2014)\end{array}$ & $\begin{array}{l}\text { Collectivist } \\
\text { Members } \\
\text { Subsample }(\mathrm{N}= \\
517) \\
\text { Individualistic } \\
\text { Members } \\
\text { Subsample }(\mathrm{N}= \\
455)\end{array}$ & $\begin{array}{c}\text { Taiwan, } \\
\text { Hong Kong, } \\
\text { and China }\end{array}$ & $\begin{array}{c}\text { Virtual } \\
\text { communities }\end{array}$ & $\begin{array}{c}\text { Extended Theory } \\
\text { of planned } \\
\text { behavior } \\
+ \\
\text { social identity } \\
\text { theory } \\
+ \\
\text { theory of collective } \\
\text { intentionality }\end{array}$ & $\begin{array}{l}\text { Subjective norm, group } \\
\text { norm, social identity, } \\
\text { anticipated } \\
\text { desires, attitude PBC, we- } \\
\text { intentions }\end{array}$ \\
\hline 45. & $\begin{array}{l}\text { Nistor et al. } \\
(2015)\end{array}$ & $\begin{array}{l}136 \text { German and } \\
\text { Romanian scholars }\end{array}$ & $\begin{array}{l}\text { Germany and } \\
\text { Romania }\end{array}$ & $\begin{array}{l}\text { communities of } \\
\text { practice (CoP) }\end{array}$ & $\begin{array}{c}\text { Sense of } \\
\text { community theory }\end{array}$ & $\begin{array}{l}\text { time in CoP, centrality in } \\
\text { CoP, and socio-emotional } \\
\text { interpersonal knowledge } \\
\text { (SEIK) }\end{array}$ \\
\hline 46. & $\begin{array}{l}\text { Gang \& } \\
\text { Ravichandran } \\
(2015)\end{array}$ & $\begin{array}{l}118 \text { experts from } \\
\text { Global Network of } \\
\text { Korean } \\
\text { Scientists and } \\
\text { Engineers } \\
\text { (KOSEN) }\end{array}$ & South Korea & $\begin{array}{l}\text { Virtual } \\
\text { communities } \\
\text { (VCs) }\end{array}$ & $\begin{array}{c}\text { Theory of reasoned } \\
\text { action } \\
+ \\
\text { Social exchange } \\
\text { theory }\end{array}$ & $\begin{array}{l}\text { Trust, anticipated } \\
\text { reciprocal relationships, } \\
\text { attitude, } \\
\text { perceived information } \\
\text { quality }\end{array}$ \\
\hline 47. & $\begin{array}{l}\text { Chang et al. } \\
(2015)\end{array}$ & $\begin{array}{l}150 \text { members of a } \\
\text { technical virtual } \\
\text { community }\end{array}$ & Taiwan & $\begin{array}{c}\text { Virtual } \\
\text { communities }\end{array}$ & $\begin{array}{c}\text { Social cognitive } \\
\text { theory } \\
+ \\
\text { Trust-commitment } \\
\text { theory } \\
+ \\
\text { Theory of planned } \\
\text { behavior } \\
\end{array}$ & $\begin{array}{l}\text { Trust, commitment, } \\
\text { knowledge self-efficacy, } \\
\text { experience }\end{array}$ \\
\hline 48. & Ye et al. (2015) & $\begin{array}{l}169 \text { from } 6 \text { online } \\
\text { knowledge } \\
\text { communities }\end{array}$ & Did not state & $\begin{array}{l}\text { Online knowledge } \\
\text { communities }\end{array}$ & $\begin{array}{c}\text { Organizational } \\
\text { support theory } \\
+ \\
\text { Social exchange } \\
\text { theory }\end{array}$ & $\begin{array}{l}\text { Pro sharing Norm, } \\
\text { information need } \\
\text { fulfillment, perceived } \\
\text { recognition from the } \\
\text { leader, perceived presence } \\
\text { of a leader, perceived } \\
\text { community support, } \\
\text { perceived leader support. }\end{array}$ \\
\hline 49. & $\begin{array}{l}\text { Yao et al. (2015) } \\
\text {. }\end{array}$ & $\begin{array}{l}222 \text { members from } \\
\text { various VC }\end{array}$ & Taiwan & $\begin{array}{c}\text { Virtual } \\
\text { communities } \\
\end{array}$ & $\begin{array}{c}\text { Social capital } \\
\text { theory }\end{array}$ & $\begin{array}{l}\text { Social capital, team } \\
\text { learning, e-loyalty }\end{array}$ \\
\hline 50. & $\begin{array}{l}\text { Cheng \& Guo } \\
(2015)\end{array}$ & $\begin{array}{l}348 \text { members of } \\
\text { Baidu Space } \\
\text { (online platform) }\end{array}$ & China & $\begin{array}{c}\text { Virtual } \\
\text { communities }\end{array}$ & $\begin{array}{c}\text { Social identity } \\
\text { theory } \\
+ \\
\text { Social capital } \\
\text { theory } \\
\end{array}$ & $\begin{array}{l}\text { Social interaction, } \\
\text { membership esteem, } \\
\text { social identity, self- } \\
\text { identity }\end{array}$ \\
\hline 51. & $\begin{array}{l}\text { Khansa et al. } \\
2015\end{array}$ & $\begin{array}{l}\text { 2,920 Yahoo! } \\
\text { Answer users }\end{array}$ & $\begin{array}{l}\text { United States } \\
\text { of America }\end{array}$ & $\begin{array}{l}\text { Online question- } \\
\text { and-answer } \\
\text { communities }\end{array}$ & Goal-setting theory & $\begin{array}{l}\text { Incentives, level of } \\
\text { membership, tenure, } \\
\text { current behaviors, prior } \\
\text { behavior }\end{array}$ \\
\hline 52. & Yen 2016 & $\begin{array}{l}201 \\
\text { teachers'/educators } \\
\text { users }\end{array}$ & Taiwan & $\begin{array}{l}\text { virtual community } \\
\text { (VC), social } \\
\text { network sites } \\
\text { (SNS), }\end{array}$ & $\begin{array}{c}\text { social capital } \\
\text { theory } \\
+ \\
\text { social identity } \\
\end{array}$ & $\begin{array}{l}\text { Trust, social interaction } \\
\text { tie, shared vision, } \\
\text { interactivity (online and } \\
\text { offline), emotional }\end{array}$ \\
\hline
\end{tabular}




\begin{tabular}{|c|c|c|c|c|c|c|}
\hline No & Authors & Sample & $\begin{array}{l}\text { Country/ } \\
\text { countries }\end{array}$ & Term used & $\begin{array}{c}\text { Theory/ theories/ } \\
\text { framework }\end{array}$ & Antecedents \\
\hline & & & & & $\begin{array}{c}\text { theory } \\
+ \\
\text { use and } \\
\text { gratification theory }\end{array}$ & $\begin{array}{l}\text { support, social identity } \\
\text { (cognitive, affective and } \\
\text { evaluative) }\end{array}$ \\
\hline 53. & $\begin{array}{l}\text { Chang et al. } \\
(2016)\end{array}$ & $\begin{array}{l}253 \text { respondents } \\
\text { from Zclub and } \\
\text { Jorsindo online } \\
\text { communities }\end{array}$ & Taiwan & $\begin{array}{c}\text { Virtual } \\
\text { communities of } \\
\text { practices (VCoPs) }\end{array}$ & $\begin{array}{c}\text { Social capital } \\
\text { theory } \\
+ \\
\text { Theory of } \\
\text { collective action }\end{array}$ & $\begin{array}{l}\text { Social interaction ties, } \\
\text { shared vision, shared } \\
\text { language, trust, } \\
\text { commitment, sense of } \\
\text { virtual community (SOVC) }\end{array}$ \\
\hline 54. & Yan et al. 2016 & $\begin{array}{l}323 \text { users of two } \\
\text { well-known } \mathrm{OHCs}\end{array}$ & China & $\begin{array}{l}\text { Online health } \\
\text { community }\end{array}$ & $\begin{array}{l}\text { Social exchange } \\
\text { theory }\end{array}$ & $\begin{array}{l}\text { Sense of self-worth, } \\
\text { perceived social support and } \\
\text { reputation enhancement, } \\
\text { face concern, cost (cognitive } \\
\text { and executional) }\end{array}$ \\
\hline 55. & $\begin{array}{l}\text { Yuan et al. } \\
(2016)\end{array}$ & $\begin{array}{l}364 \text { airline } \\
\text { passenger online } \\
\text { community }\end{array}$ & China & $\begin{array}{l}\text { Online travel } \\
\text { communities }\end{array}$ & $\begin{array}{c}\text { consumer } \\
\text { psychology } \\
\text { literature } \\
\quad+ \\
\text { Technology } \\
\text { Acceptance } \\
\quad \text { Model } \\
\end{array}$ & $\begin{array}{l}\text { Innovativeness, subjective } \\
\text { knowledge, perceived ease } \\
\text { of use, perceived usefulness }\end{array}$ \\
\hline 56. & $\begin{array}{l}\text { Hau \& Kang } \\
(2016) \text {. }\end{array}$ & $\begin{array}{l}140 \text { online } \\
\text { community user }\end{array}$ & South Korea & $\begin{array}{l}\text { Online user } \\
\text { community }\end{array}$ & $\begin{array}{c}\text { lead user theory } \\
+ \\
\text { social capital } \\
\text { theory } \\
+ \\
\text { social cognitive } \\
\text { theory }\end{array}$ & $\begin{array}{l}\text { Social capital (tie, trust, } \\
\text { goals), lead users, perceived } \\
\text { behavioral control (self- } \\
\text { efficacy, controllability) }\end{array}$ \\
\hline 57. & $\begin{array}{l}\text { Feng and Ye } \\
(2016)\end{array}$ & $\begin{array}{l}169 \text { from online } \\
\text { English forum }\end{array}$ & China & $\begin{array}{l}\text { Online knowledge } \\
\text { community }\end{array}$ & $\begin{array}{c}\text { equity theory } \\
+ \\
\text { Social Identity } \\
\text { explanation of De- } \\
\text { individuation } \\
\text { Effects (SIDE) } \\
\end{array}$ & $\begin{array}{l}\text { Community norm, } \\
\text { indebtedness, perceived } \\
\text { anonymity, intention to } \\
\text { reciprocate }\end{array}$ \\
\hline 58. & $\begin{array}{l}\text { Zhang et al. } \\
(2017 \mathrm{a})\end{array}$ & $\begin{array}{l}443 \text { sample from } \\
\text { three famous } \\
\text { online health } \\
\text { communities }\end{array}$ & China & $\begin{array}{l}\text { Online health } \\
\text { communities }\end{array}$ & Motivation theory & $\begin{array}{l}\text { Reputation, reciprocity, self- } \\
\text { efficacy, altruism, empathy }\end{array}$ \\
\hline 59. & Liao (2017). & $\begin{array}{ll}176 & \text { college } \\
\text { students }\end{array}$ & Taiwan & $\begin{array}{c}\text { Virtual } \\
\text { communities }\end{array}$ & $\begin{array}{l}\text { Social influence } \\
\text { theory }\end{array}$ & $\begin{array}{l}\text { cognitive benefits, social } \\
\text { integrative benefits, } \\
\text { personal integrative } \\
\text { benefits, and hedonic } \\
\text { benefits }\end{array}$ \\
\hline 60. & $\begin{array}{l}\text { Alsharo et al. } \\
(2017)\end{array}$ & $\begin{array}{l}193 \text { respondents } \\
\text { from } \\
\text { LindkedIN.com }\end{array}$ & Jordan & Virtual team & $\begin{array}{c}\text { Social capital } \\
+ \\
\text { Social exchange }\end{array}$ & $\begin{array}{l}\text { Trust, collaboration, team } \\
\text { effectiveness }\end{array}$ \\
\hline 61. & $\begin{array}{l}\text { Zhang et al. } \\
(2017 b)\end{array}$ & $\begin{array}{lr}516 & \text { college } \\
\text { students } & \text { (VIC } \\
\text { users') } & \end{array}$ & China & $\begin{array}{l}\text { Virtual innovation } \\
\text { community (VIC) }\end{array}$ & $\begin{array}{c}\text { social cognitive } \\
\text { theory } \\
+ \\
\text { social exchange } \\
\text { theory }\end{array}$ & $\begin{array}{l}\text { Self-efficacy, hope, } \\
\text { optimism, tenacity, material } \\
\text { reward, reciprocal } \\
\text { relationship }\end{array}$ \\
\hline 62. & $\begin{array}{l}\text { Park and } \\
\text { Gabbard (2018) }\end{array}$ & 141 scientist & $\begin{array}{l}\text { United States } \\
\text { of America }\end{array}$ & $\begin{array}{l}\text { Virtual } \\
\text { community }\end{array}$ & $\begin{array}{l}\text { Social exchange } \\
\text { theory }\end{array}$ & $\begin{array}{l}\text { Reciprocal benefit, } \\
\text { anticipated relationship, } \\
\text { reputation, altruism, fear of } \\
\text { losing one's value }\end{array}$ \\
\hline 63. & $\begin{array}{l}\text { Pirkkalainen et } \\
\text { al } 2018\end{array}$ & 205 respondents & Finland & $\begin{array}{l}\text { Open innovation } \\
\text { communities }\end{array}$ & $\begin{array}{l}\text { psychological } \\
\text { ownership theory }\end{array}$ & $\begin{array}{lr}\begin{array}{l}\text { Personal } \\
\text { expectation, }\end{array} & \begin{array}{r}\text { outcome } \\
\text { organizational }\end{array} \\
\text { innovativeness, } & \text { previous } \\
\text { online KS } & \text { experience, } \\
\text { commitment, openness to } \\
\text { experience, psychological } \\
\text { ownership }\end{array}$ \\
\hline 64. & Guan et al. 2018 & $\begin{array}{l}1599 \text { Q\&A online } \\
\text { users }\end{array}$ & China & $\begin{array}{l}\text { online Q\&A } \\
\text { communities }\end{array}$ & $\begin{array}{c}\text { Social capital } \\
\text { theory } \\
+ \\
\text { social exchange } \\
\end{array}$ & $\begin{array}{lr}\text { Identity-based } & \text { trust, social } \\
\text { feedback, } & \text { identity } \\
\text { communication, } & \text { social } \\
\text { exposure, norms } & \text { of } \\
\end{array}$ \\
\hline
\end{tabular}




\begin{tabular}{|c|c|c|c|c|c|c|}
\hline No & Authors & Sample & $\begin{array}{l}\text { Country/ } \\
\text { countries }\end{array}$ & Term used & $\begin{array}{c}\text { Theory/ theories/ } \\
\text { framework }\end{array}$ & Antecedents \\
\hline & & & & & $\begin{array}{c}\text { theory } \\
+ \\
\text { social identity } \\
\text { theory } \\
+ \\
\text { social cognitive } \\
\text { theory }\end{array}$ & reciprocity \\
\hline 65. & $\begin{array}{lll}\text { Xiong } & \text { et } & \text { al. } \\
2018 & & \end{array}$ & $\begin{array}{l}666 \text { of online } \\
\text { communities (Baid } \\
\text { Bar) }\end{array}$ & China & $\begin{array}{c}\text { Virtual } \\
\text { communities }\end{array}$ & $\begin{array}{c}\text { Social capital } \\
+ \\
\text { Social identity } \\
+ \\
\text { Social exchange }\end{array}$ & $\begin{array}{l}\text { Status of opinion leader, } \\
\text { self-identity, reciprocity, } \\
\text { social interaction tie }\end{array}$ \\
\hline 66. & $\begin{array}{l}\text { Kang } \\
(2018\end{array} \quad$ et al. & 359 respondents & $\begin{array}{l}\text { United States } \\
\text { of America }\end{array}$ & $\begin{array}{c}\text { Virtual } \\
\text { communities }\end{array}$ & $\begin{array}{l}\text { Social capital } \\
\text { theory }\end{array}$ & $\begin{array}{lr}\text { Anonymity, virtual network } \\
\text { connectivity, } \\
\text { network closeness }\end{array}$ \\
\hline 67. & $\begin{array}{l}\text { Kumi and } \\
\text { Sabherwal } \\
(2018)\end{array}$ & $\begin{array}{l}144 \text { users from } \\
\text { two online } \\
\text { communities }\end{array}$ & $\begin{array}{l}\text { United States } \\
\text { of America }\end{array}$ & $\begin{array}{l}\text { online discussion } \\
\text { communities } \\
\text { (ODCs) }\end{array}$ & Social identity & $\begin{array}{lr}\text { Intrinsic } & \text { motivation, } \\
\text { extrinsic motivations, social } \\
\text { identity } \\
\text { affective, } \\
\text { satisfaction with community }\end{array}$ \\
\hline 68. & Hao et al. (2019 & $\begin{array}{l}219 \text { virtual team } \\
\text { members }\end{array}$ & China & Virtual team & $\begin{array}{c}\text { personality traits } \\
\text { theories } \\
+ \\
\text { Job Characteristics } \\
\text { Model } \\
+ \\
\text { Job Demands- } \\
\text { Resources } \\
\text { Model } \\
+ \\
\text { social cognitive } \\
\text { theory }\end{array}$ & $\begin{array}{l}\text { Conscientiousness, } \\
\text { demands of skill variety, } \\
\text { knowledge sharing self- } \\
\text { efficacy }\end{array}$ \\
\hline
\end{tabular}

\section{Theories in Virtual community studies}

There were various theories used to understand member $\mathrm{KS}$ in VC. As the VC platform involve social interaction among members, the majority of researchers applied theories related to social factors that can ultimately understand the antecedents of member's behavior. In this section, a list of mostly used theories are discussed:

\section{Theory of planned behavior and reasoned action}

This theory is probably the most used in understanding $\mathrm{KS}$ behavior. It was first developed by Ajzen and Fishbein (1975) as theory of reasoned action. An individual intention towards a behavior is determined by the attitude and subjective norm. In 1991, Ajzen had extended the theory of reasoned action into the theory of planned behavior by introducing perceived behavioral control as the third independent variable. Perceived behavioral control explains the ability of a person to perform the behavior in question. The use of TPB in VC studies enables scholars to understand the behavior of members to share depend on the personal attitude, perception of other people towards the behavior (subjective norm), and the perceived ability of the person to perform it. Studies that applied the theory of reasoned action were done by Shu and Chuang (2011) and Lin and Huang (2013), while Gang and Ravichandran (2015) integrated the theory with social exchange theory. Various studies had used TPB in VC KS studies. This includes Erden et al. (2012) and Chen et al. (2012). Some studies integrated this theory with other relevant theories by incorporating social-related factors and extending the TPB model (Jeon et al. 2011b; Cho et al. 2010; Tsai\& Bagozzi 2014; Chang et al. 2015).

\section{Technology Acceptance Model (TAM)}

TAM is a model that explains individual behavior that is dependent on an individual's attitude in executing that specific behavior. It evaluates each of that outcome as a function of one's belief on the expectation in performing such behavior $(\mathrm{Ku}, 2012)$. the model constitutes the concept of usefulness and perceived ease of use (Davis, 1989). Perceived usefulness is a person's perception that using technology can bring benefits and enhance their application performance. Perceived ease of use is the ability to use such technology without having any difficulty (Papadopoulos et al., 2013). The review found thatfour 
relevant studies applied the technology acceptance model (Hung \& Cheng, 2013; Ku, 2012; Papadopoulos et al., 2013; Yuan et al., 2016).

\section{Social Capital Theory}

The social capital theory explains the network of relationships that are created among individuals or a group of people with the set of resources within it. This system would have enhanced and positively impact interpersonal KS among the members (Chiu et al. 2006). Nahapiet and Ghoshal (1998) described social capital theory in three perspectives, based on KS in communities. The structural capital (nodes that are interconnected, creating a pattern), relational capital (leveraging and creating assets through connection), and cognitive capital (systems of meaning among members that produce resources by having shared representation and interpretations). Among the factors identified in social capital are trust, recognition, common language, and shared vision. Among studies that practiced only social capital theory was Yao et al. (2015). In practice, social capital theory usually combined with other theories in assessing members of virtual communities KS behaviour. Many studies integrated social capital with either social cognitive theory, social identity, social exchange and other related theories (Chiu et al. 2006; Shan et al. 2013; Zeng et al. 2014; Tseng \& Kuo, 2014; Hau \& Kang 2016; Cheng \& Guo, 2015, Yen, 2016; Chang et al., 2016; Al-Sharo et al., 2017; Guan et al. 2018). A theory related to social capital is the theory of collective action. The theory explained how problems should be avoided, arising from the existence of conflicting incentives. It suggests individuals engage with action in context due to social capital (Coleman, 1990). The study that applied this theory was Chang et al., (2016).

\section{Social Cognitive Theory}

The social cognitive theory comprises three factors, personal, environment, and behavior that play essential roles in influencing individual interactivity (Hsu et al. 2007). The social cognitive theory explained that individual personal factors interact with the behavior and environmental aspect, which ends up with triadic reciprocity ( $\mathrm{Lu}$ et al. 2013). Among the determinants in personal factors are self-efficacy and outcome expectations, as both can predict a person's behavior (Bandura, 1997). While trust and altruism are considered as an environmental factor as it can influence personal characteristics and the behavior itself (Papadopoulos et al., 2013). Others had considered the norm of reciprocity as factors in the environment while perceived relative advantage and perceived compatibility as personal factors (Lin et al., 2009; Chen and Hung 2010), There is one study that tested on several individual factors such as hope, optimism, and tenacity (Zhang et al. 2017a). The social cognitive theory had been applied solely in many studies (Hsu et al., 2007; Lin et al., 2009; Chen \& Hung, 2010; Xu et al., 2012; $\mathrm{Lu}$ et al., 2013). There are also many studies had combined and integrated with other theories (Chiu et al. 2006; Cho et al., 2010; Shan et al., 2013; Papadopoulos et al. 2013; Zeng et al., 2014; Tseng \& Kuo, 2014; Chang et al., 2015; Hau \& Kang, 2016; Zhang et al., 2017b; Guan et al., 2018 Hao et al., 2019).

\section{Social Identity Theory}

Based on social identity theory, the characteristic of a person determines their sharing behavior rather than the distinctive feature, according to their social identification (Turner, 1982). This theory is rooted in personal belongings to a group. It is an individual-based perception that defined the team within a group membership. Social identity differentiates a person and other members of a group from other group members (Kim et al. 2011). Social identity consists of three main domains of the interactive process of psychology: cognitive, evaluative, and affective (Kumi \& Sabherwal, 2018). Personal identification within social groups enables members to develop selfesteem and positively influence attitudes and behaviors. The affective domain plays a role in the group's emotional attachment. Evaluative, on the other hand, is vital to personal selfesteem as an outcome of association with groups. Cognitive in self-identity is the creation of awareness belonging to a group and having an objective in pursuing goals. These three main attributes of social identity explain how individual develop identities and hence leads to attitude and behaviors (Tajfel, 1978).Studies that applied social identity theory include Kim et al. (2011), Ku (2012), and Zhao et al. (2012). Studies that integrated identity theory with other theory include social presence (Shen et al., 2010) social 
cognitive and social exchange and social capital (Zeng et al. 2014; Cheng \& Guo; Guan et al. 2018) and extended theory of planned behavior (Tsai \& Bagozzi, 2014).

\section{Social Exchange Theory}

The social exchange was developed in explaining a non-contractual relationship between individuals (Staples \& Webster, 2008). Social exchange in VC studies posits that the engagement between individuals in online communities enables them to receive some form of benefit (Phang et al. 2009). VC serves as a platform for users to contribute and receive different information and resources from various sources. These resources are a form of social exchange, either tangible or intangible. During the exchange, it may constitute cost or benefits. The use of social exchange theory may facilitate scholars to assess which dimension or factors that are highly perceived by individual either in knowledge contribution or act of seeking. Social exchange theory explains that the factors that lead the individual towards social interaction are based on the expectation that he or she would receive social rewards, such as respect, status, and approval (Liao et al. 2013). The level of interaction is based on cost and benefits consideration. If the benefits are more than the cost, the chance for them to engage in such interaction is high. Among the crucial factors in social exchange theory is trust. For the social exchange to be realized, reciprocation among the exchange members should be rooted in trust (Blau, 1964). This is to ensure a complete exchange to note the perceived obligation and strengthen trustworthiness among members in VC.

Literature shows that plenty of studies had applied this theory (Staples \& Webster, 2008; Liao et al. 2013; Yan et al., 2016; Park \& Gabbard, 2018). Some other studies had combined with different theories, such as the theory of reasoned action (Gang \& Ravichandran, 2015), the theory of social capital, identity and cognitive (Guan et al., 2018; Xiong et al. 2018) and value theory (Phang et al., 2009). An extension of the social exchange theory is value theory. It explained that an individual is attached to different values based on objects or concepts that can fulfill their requirement (Harper, 1974). Studies that applied value theory were Phang et al. (2009) and Lai \& Chen (2014). Another theory that is similar to the social exchange theory is rational action theory. According to rational action theory, if a person perceived that there is benefit from a particular behavior, it will be more likely for them to engage with the said behavior (Hau et al. 2013). In line with this theory, expected benefits such as organizational reward, reciprocity, and enjoyment would encapsulate one to share in an online platform.

Meanwhile, equity theory corresponds to the perception and request of fairness/equity of individuals concerning a relationship (Cohen \& Greenberg, 1982). According to Feng and Ye (2016), equity theory is the assessment of an individual in social exchange. It includes the ratio of the input and output from a relationship. It also has other person ration of the production from their input. Equity theory suggests that a healthy relationship between individual existed when each of them perceived that their output is equal to what they have provided in the input, suggesting fair as key to KS.Organizational support theory is also an extension of the social exchange theory. The theory proposes that there two categories of perception (organizational support and supervisor support) that constitute one behavior within an organization (Eisenberger et al., 2002). It also refers to the general belief behold by an individual concerning how the organization values their contribution and welfare is being taken care of (Eisenberger et al., 1986).

\section{Expectancy Disconfirmation Theory (EDT)}

Another theory in VC KS behavior is expectancy disconfirmation theory (EDT) that was proposed by Oliver (1980). It explained that person repurchase intentions are depending on their satisfaction. This theory explains an individual continuance to share knowledge based on their satisfaction as the main factor (Jin et al., 2013). It is also known as expectancy confirmation theory. Satisfaction, on the other hand, is depending on one prepurchase expectation and post-purchase disconfirmation of expectations. In 1993, EDT was revived by including affection, performance, and equity as the antecedents of customer satisfaction and repurchase intention. This theory work by the customers resulting in expectations positively confirmed (perceived performance more than expectation), confirmed (perceived 
performance same as expectation), and negatively confirmed (perceived performance less than expectation). Customer's or user's expectation is the basis of evaluating satisfaction. In an event where confirmation occurs, the satisfaction will fall either lower or more than the expectation. A common theme found in this theory, regardless of its application, is the disconfirmation size and direction. Consumers would be satisfied in the case of positive disconfirmation and dissatisfied when negative disconfirmation occurs (Venkatesh \& Goyal, 2010). In the VC context, members would have a certain expectation in using the platform to search for knowledge. Their future participation will depend on their satisfaction on whether their participation would enable them to receive relevant knowledge based on their needs. Studies in VC that had applied this theory were Chiu et al. (2011) and Jin et al. (2013). Chiu et al. (2011) had integrated with justice theory and social network factors.

\section{Motivation Theory}

Three theories are based on motivation: motivation theory, self-determination theory, and expectancy theory of motivation. Motivation theory was developed to understand human behaviorderived from the expectation of beneficial outcomes (Zhang et al., 2017a). The two main motivation is intrinsic and extrinsic. Self-determination theory (SDT) is a theory explaining human motivation that is concerned with tendencies of one's inherent growth and the needs of their psychology. These two main domains have a direct effect on their self-motivation (Yoon and Rolland, 2012). On the other hand, the expectancy theory of motivation is based on the perceived consequences factor that relies on the action and value of each consequence (Vroom, 1964). The perceived consequence is known as the chances of a specific consequence that would like to happen as a result of a behavior.

\section{Use and Gratification Theory (U\&G theory)}

U\&G theory originated from the field of communication, where scholars apply it to determine why members engage in the community as a tool in fulfilling their needs (Chen et al. 2013; Mairaru et al. 2019). Katz et al. (1974) posited that the U\&G theory was introduced to understand the user's application of media. How the users take the initiative, rather than being a free rider in receiving the message and manipulating medium in searching for an information meeting their needs. As the fast increment of internet usage since the last two decades and a rapid level of interactivity compared to conventional media has paved the way for U\&G theory to assess internet use motivations. The theory has been adapted in user acceptance of instant messaging, the internet, emails, electronic bulletin boards, and VC (Yen, 2016). With the development of the internet, the user's acceptance of the theory has been empowered due to easy conversion from one medium to another (Xu et al., 2012).

U\&G is based on three dimensions: process, socialization, and content (Peters et al. 2007). These three dimensions can be further illustrated as entertainment (process), social needs (socialization), and information (content). The user's motivation to participate in acquiring knowledge in VC is different. Knowledge required in professional forums is perceived to be more critical than social-based VC, thus focusing on the content dimension. While members in social-based, their weigh is more on the social needs and entertainment aspect. In studying VC KS behavior, these three dimensions would vary depends on the platform basis and the community involved. Studies applying U\&G theory were Chiu et al., (2013) and Yen (2016).

\section{Other theories}

Other relevant theories used in this study are the Triandis model (Jeon et al., 2011a; Tamjidyamcholo et al., 2014). This theory explains an individual towards behavior in question. The behavior is influenced by facilitating factors that can positively impact, such as perceived consequences, social factors, and affect. Another critical theory is the sense of community theory. It was first formulated by McMillan and Chavis (1986), which was further enhanced by McMillan (1996). The purpose of community theory is described as the belonging feelings and feeling that the group members are matter to one another, having a shared faith among members. Each member needs to commit to meet the member's need. While an advanced definition of this theory is described as a "feeling of belonging spirit, having trust feeling on the structure of authority. Awareness and mutual benefit that come from being together with the 
spirit that exhibits from shared experience, preserved as art" (McMillan, 1996, p. 315). The study that applied a sense of community theory was Nistor et al., (2015). Other theories applied were self-perception theory (Yan et al., 2013), goal setting theory (Khansa et al., 2015), lead user theory (Hau and Kang, 2016), social influence theory (Liao, 2017), attribution and self-presentation theory (Ma \& Agarwal, 2007)

\section{DISCUSSION}

Understanding the theories used in $\mathrm{VC}$ members, KS is vital. A majority of studies focused on the aspect of social within all the theories presented; understandably, social factorsare deemed to be of the utmost importance. Even the theory of planned behavior and reasoned action is based on human relationships with one another on the aspect of the subjective norm (Erden et al., 2012). Almost all the major studies have used social-related theories directly in their studies (social exchange, social identity, social cognitive, and social capital). Even though other studies did not apply a direct social theory, mostly other theories have embedded a social foundation underlying construct or having social-relational constructs such as trust, reputation, norm of reciprocity, and enjoyment in helping others. These factors are so important in VC studies as interaction with other people may lead to trustworthiness that builds up from such a relationship.

Trust is the most studies factor in KS literature (Bandura, 1997). The importance of trust in understanding VCmembers $\mathrm{KS}$ is deemed crucial as sharing on the internet comes with the possibility of risk and liability. Trust, together with commitment, is considered to be supported by the feeling of belongingness (Chang et al. 2016, Capello and Fagian, 2005). The concept of trust is well documented within three theories, namely social capital, social identity, and social exchange. Documented from this review, the majority of studies applied the construct of trust either directly from the three theories or by having the trust construct as an indirect factor. Multiple studies applied theories that are based on trust by nature such as applying trust in multiple dimension (Chai \& Kim, 2010), trust-building factors (Hsu et al., 2011), Social factors which include trust (Majewski et al., 2011) and trust-commitment theory (Chang et al., 2015).
The three most cited categories within the trust domain are ability, integrity, and benevolence (Zeng et al., 2014). Meanwhile, Usoro et al.(2007)termed the ability to trust as competence-based trust. Integrity is regarded as the expectation of an individual that everyone within VC is following a prescribed general set of rules, principles, and values (Chiu et al., 2006). Benevolence is a preference to commit to a kind and charitable act. Meanwhile, competence or ability is known as the activity based on one qualification, skills, and training (Usoro et al., 2007). These three domains are distinct among each other, but the result shows that they are empirically inseparable. For a proper KS to be realized, all three dimensions of trust should be inculcated. Apart from the trust domain, there have been researchers that studied trust within a different aspect. Such as work by Hsu et al. (2011) that studied trust in members and trust in the system. This is the only study that focuses on the trust in the system. It is deemed vital in such a way when every member of VC can be trustworthiness, but the system might jeopardize a member's personal information. If the system is compromised, data can be leaked to the wrong hand, to the extent used for criminal activities. The two different categories of trust have made an understanding of trust in different aspects of KS via VC. On the other hand, Hau et al. (2013) have adopted a trusted domain in measuring the different types of behavior of sharing based on tacit and explicit knowledge. It was identified that trust and risk profiles of users are different based on the type of knowledge they share (tacit and explicit) (Becerra et al., 2008).

Social exchange theory has a very significant impact on the study of VC members KS. This theory is based on the perceived benefits and the return of sharing behaviors of members in VC. The human being would want something in return when they contribute and participate. As this basis, the theory has led to other related or derivation from a social exchange such as respect, approval, and status (Liao et al., 2013). This social reward would be the main reason whether members are willing to share their knowledge freely. The theory perceived that human would weigh the cost and benefits before engaging in any social interaction. Phang et al. (2009) and Lai \& Chen (2014) applied value theory that suggests members value realizing from VC that 
confides on participation in knowledge seeking and contribution. According to Harper (1974), a different type of profession and group value knowledge differently which lead to their specific behavior. Professionals may value VC as a source of knowledge within their specific filed while it is different from a socially-oriented VC. A VC that focuses on social may regard it as a platform for entertainment. Meanwhile, in equity theory (a similar theory to social exchange) suggest that fairness or equity should adhere when individual engage in any relationship. The theory suggests that when one provides certain input in a relationship, he or she should attain the same amount of output. Other theories having a similar concept to social exchange are rational action theory and organizational support theory. It can be understood from the literature that having much exchange within the community is vital in ensuring that knowledge can be circulated within the VC network. Factors related to selfattainment must be attached within the scope of KS. This attainment is considered not only in virtual setting but in a physical KS environment.

\section{FUTURE WORK}

Future avenues should see in the context of personalities that are significant towards VC member's KS. From the 68 studies, no studies hadapplied the big five personalities that are commonly used by scholars in organizational studies. Despite that were few studies undertaken on a broader aspect personality (Chu et al., 2014) and preferably on some specific personality of the big five such as conscientiousness (Fang \& Chiu, 2010; Hao et al., 2019) and openness to experience (Yu et al., 2010). From these extensive and latest studies on KS behavior on VC, it can be seen that personality traits were somewhat neglected and received little attention. By understanding which traits are having the most impact on KS behavior, scholars and practitioners can make use of the VC platform to be the best possible mean in ensuring that knowledge can be disseminated. For example, it may be postulated that those who are high in extroversion and conscientiousness would have no problem communicating and sharing knowledge due to their inclination towards open interaction and enjoy helping others. On the other hand, a person high in neuroticism might be more cautious, and having a calculative measure to share and received information either from peers or strangers. All the social-related factors can be related to individual personality traits that can provide meaningful insight into organizational behavior studies such as in VC.

With extensive studies on $\mathrm{VC}$, there have been many terms used in describing individual online knowledge participation. Another term used by Hsseinoiun et al. (2018) is big data community, focusingon how scholars are actively participating in information and knowledge retrieval from big data. The domain focused on knowledge quality, system quality, use, user satisfaction, and community success. With the enhanced development of industrial revolution 4.0, the need to engage within big data analytics would see as a significant leap towards advanced technological adoption. As younger members participate in $\mathrm{VC}$, the perceived ability to apply advanced tools in VC is deemed appropriate. Those who are not able to commit to using recent technology would be left behind and thus unable to make benefits from it. Future work should look into using the theory of acceptance model with the application of industrial revolution software and tools. Furthermore, the use of big data and data analytics should pave the way for VC moderators and stakeholders in acquiring useful information that can further improve the function of VC in the near future.

\section{CONCLUSION}

VC has provided the best possible environment for the communities to engage in KS activities. As the world develops even further, people would prefer to attain information and knowledge within a short period. The availability of $\mathrm{VC}$ realizes this rapid information retrieval. More and more professional individuals from various professions and socially orientedcommunities have used VC to acquire or share their knowledge. This paper has managed to review 68 studies within the 13-year period (2006-2019) that has conducted KS studies within the scope of VC. Most of the studies were focusing on the related social theories that involved much domain of social variables such as trust, reputation, norm of reciprocity, enjoyment in helping, and many others. All these antecedents are within the scope of main theories available within VC literature encompassing the theory of planned behavior, 
technology acceptance model, social capital theory, social identity theory, social exchange theory, expectancy disconfirmation theory, and use \& gratification theory. Other related theories and antecedents had also been briefly discussed throughout this review process.

\section{REFERENCES}

Ajzen, I. and Fishbein, M. (1975), Belief, Attitude, Intention and Behaviour, an Introduction to Theory and Research, Addison-Wesley Publication, New York, NY.

Ajzen, I. (1991). The theory of planned behavior. Organizational behavior and human decision processes, 50(2), 179-211.

Akhavan, P., Hosseini, S. M., Abbasi, M., \& Manteghi, M. (2015). Knowledge-sharing determinants, behaviors, and innovative work behaviors: an integrated theoretical view and empirical examination. Aslib Journal of information management, 67(5), 562-591.

Al-Kurdi, O., El-Haddadeh, R., \& Eldabi, T. (2018). Knowledge sharing in higher education institutions: a systematic review. Journal of Enterprise Information Management, 31(2), 226-246.

Alsharo, M., Gregg, D., \& Ramirez, R. (2017). Virtual team effectiveness: The role of knowledge sharing and trust. Information \& Management, 54(4), 479-490.

Bandura, A., 1997. Self-efficacy: The Exercise of Control. Freeman, New York, NY.

Becerra, M., Lunnan, R., \& Huemer, L. (2008). Trustworthiness, risk, and the transfer of tacit and explicit knowledge between alliance partners. Journal of Management Studies, 45(4), 691-713.

Blau, P. (1964). Exchange and power in social life. New York, NY: Wiley.

Bolisani, E., \& Scarso, E. (2014). The place of communities of practice in knowledge management studies: a critical review. Journal of Knowledge Management, 18(2), 366-381.

Capello, R., \& Faggian, A. (2005). Collective learning and relational capital in local innovation processes. Regional Studies, 39(1), 75-87.

Chai, S., \& Kim, M. (2010). What makes bloggers share knowledge? An investigation on the role of trust. International journal of information management, 30(5), 408-415.
Chang, H. H., Hsieh, P. H., \& Fu, C. S. (2016). The mediating role of sense of virtual community. Online Information Review, 40(7), 882-899.

Chang, C. M., Hsu, M. H., \& Lee, Y. J. (2015). Factors influencing knowledge-sharing behavior in virtual communities: A longitudinal investigation. Information Systems Management, 32(4), 331-340.

Chen, C. J., \& Hung, S. W. (2010). To give or to receive? Factors influencing members' knowledge sharing and community promotion in professional virtual communities. Information \& management, 47(4), 226-236.

Chen, I. Y., \& Chen, N. S. (2009). Examining the factors influencing participants' knowledge sharing behavior in virtual learning communities. Journal of Educational Technology \& Society, 12(1).

Chen, G. L., Yang, S. C., \& Tang, S. M. (2013). Sense of virtual community and knowledge contribution in a P3 virtual community: Motivation and experience. Internet Research, 23(1), 4-26.

Cheng, Z. C., \& Guo, T. C. (2015). The formation of social identity and self-identity based on knowledge contribution in virtual communities: An inductive route model. Computers in Human Behavior, 43, 229-241.

Chiu, C. M., Hsu, M. H., \& Wang, E. T. (2006). Understanding knowledge sharing in virtual communities: An integration of social capital and social cognitive theories. Decision support systems, 42(3), 1872-1888.

Chiu, C. M., Wang, E. T., Shih, F. J., \& Fan, Y. W. (2011). Understanding knowledge sharing in virtual communities: An integration of expectancy disconfirmation and justice theories. Online Information Review, 35(1), 134-153.

Cho, H., Chen, M., \& Chung, S. (2010). Testing an integrative theoretical model of knowledgesharing behavior in the context of Wikipedia. Journal of the American Society for Information Science and Technology, 61(6), 1198-1212.

Chu, M. T., KrishnaKumar, P., \& Khosla, R. (2014). Mapping knowledge sharing traits to business strategy in knowledge based 
organisation. Journal of Intelligent Manufacturing, 25(1), 55-65.

Cohen, R. L., \& Greenberg, J. (1982). The justice concept in social psychology. In Equity and justice in social behavior (pp. 1-41).

Coleman, J. S. (1990). Foundations of Social Theory. Social Forces, 69(2), 625-633.

Davis, F. D. (1989). Perceived usefulness, perceived ease of use, and user acceptance of information technology. MIS Quarterly, 13(3), 319-340.

Djelantik, S. (2019). Islamic State and the Social Media in Indonesia. Journal of Content, Community \& Communication Amity School of Communication, 9(2), 147-155

Eisenberger, R., Huntington, R., Hutchison, S., \& Sowa, D. (1986). Perceived organizational support. Journal of Applied psychology, 71(3), 500.

Eisenberger, R., Stinglhamber, F., Vandenberghe, C., Sucharski, I. L., \& Rhoades, L. (2002). Perceived supervisor support: Contributions to perceived organizational support and employee retention. Journal of applied psychology, 87(3), 565.

Erden, Z., Von Krogh, G., \& Kim, S. (2012). Knowledge sharing in an online community of volunteers: the role of community munificence. European Management Review, 9(4), 213-227.

Fang, Y. H., \& Chiu, C. M. (2010). In justice we trust: $\quad$ Exploring knowledge-sharing continuance intentions in virtual communities of practice. Computers in Human Behavior, 26(2), 235-246.

Fauzi, M. A., Nya-Ling, C. T., Thurasamy, R., \& Ojo, A. O. (2018). An integrative model of knowledge sharing in Malaysian Higher Learning Institute. Kybernetes, 47(5), 10311052.

Fauzi, M. A. (2019). Knowledge sharing in Asia Pacific via virtual community platform: a systematic review. Int. J. Web Based Communities, 15(4), 368-394

Feng, Y., \& Ye, H. J. (2016). Why do you return the favor in online knowledge communities? A study of the motivations of reciprocity. Computers in Human Behavior, 63, 342-349.

Gang, K., \& Ravichandran, T. (2015). Exploring the determinants of knowledge exchange in virtual communities. IEEE Transactions on Engineering Management, 62(1), 89-99.

Guan, T., Wang, L., Jin, J., \& Song, X. (2018). Knowledge contribution behavior in online Q\&A communities: An empirical investigation. Computers in Human Behavior, 81, 137-147.

Hao, Q., Yang, W., \& Shi, Y. (2018). Characterizing the relationship between conscientiousness and knowledge sharing behavior in virtual teams: an interactionist approach. Computers in Human Behavior.

Harper, F. A. (1974) An Introduction to Value Theory. Calif.: Institute for Humane Studies, Inc.

Hau, Y. S., Kim, B., Lee, H., \& Kim, Y. G. (2013). The effects of individual motivations and social capital on employees' tacit and explicit knowledge sharing intentions. International Journal of Information Management, 33(2), 356-366.

Hau, Y. S., \& Kang, M. (2016). Extending lead user theory to users' innovation-related knowledge sharing in the online user community: the mediating roles of social capital and perceived behavioral control. International Journal of Information Management, 36(4), 520-530.

Hsseinoiun, S., Abdullah, R., Jusoh, Y. Y., \& Jabar, M. (2018, March). Information System Success and Knowledge Grid Integration in Facilitating Knowledge Sharing Among Big Data Community. In 2018 Fourth International Conference on Information Retrieval and Knowledge Management (CAMP) (pp. 1-5). IEEE.

Hsu, M. H., Ju, T. L., Yen, C. H., \& Chang, C. M. (2007). Knowledge sharing behavior in virtual communities: The relationship between trust, self-efficacy, and outcome expectations. International journal of human-computer studies, 65(2), 153-169.

Hsu, M. H., Chang, C. M., \& Yen, C. H. (2011). Exploring the antecedents of trust in virtual communities. Behaviour \& Information Technology, 30(5), 587-601.

Hung, S. W., \& Cheng, M. J. (2013). Are you ready for knowledge sharing? An empirical study of virtual communities. Computers \& Education, 62, 8-17. 
Jeon, S., Kim, Y. G., \& Koh, J. (2011a). An integrative model for knowledge sharing in communities-of-practice. Journal of knowledge management, 15(2), 251-269.

Jeon, S. H., Kim, Y. G., \& Koh, J. (2011b). Individual, social, and organizational contexts for active knowledge sharing in communities of practice. Expert Systems with applications, 38(10), 12423-12431.

Jin, X. L., Zhou, Z., Lee, M. K., \& Cheung, C. M. (2013). Why users keep answering questions in online question answering communities: A theoretical and empirical investigation. International Journal of Information Management, 33(1), 93-104.

Kang, K., Kwak, M., \& Shin, S. K. (2018). A Calculus of Virtual Community Knowledge Intentions: Anonymity and Perceived Network-Structure. Journal of Computer Information Systems, 58(2), 110-121.

Katz, E. (1974). Utilization of mass communication by the individual. The uses of mass communications: Current perspectives on gratifications research, 19-32.

Kim, H. W., Zheng, J. R., \& Gupta, S. (2011). Examining knowledge contribution from the perspective of an online identity in blogging communities. Computers in Human Behavior, 27(5), 1760-1770.

$\mathrm{Ku}$, E. C. (2012). Distributed fascinating knowledge over an online travel community. International Journal of Tourism Research, 16(1), 33-43.

Kumi, R., \& Sabherwal, R. (2018). Knowledge sharing behavior in online discussion communities: Examining behavior motivation from social and individual perspectives. Knowledge and Process Management.

Lai, H. M., \& Chen, T. T. (2014). Knowledge sharing in interest online communities: A comparison of posters and lurkers. Computers in Human Behavior, 35, 295-306.

Liao, C., To, P. L., \& Hsu, F. C. (2013). Exploring knowledge sharing in virtual communities. Online Information Review, 37(6), 891-909.

Liao, T. H. (2017). Developing an antecedent model of knowledge sharing intention in virtual communities. Universal Access in the Information Society, 16(1), 215-224.
Lin, M. J. J., Hung, S. W., \& Chen, C. J. (2009). Fostering the determinants of knowledge sharing in professional virtual communities. Computers in Human Behavior, 25(4), 929-939.

Lin, F. R., \& Huang, H. Y. (2013). Why people share knowledge in virtual communities? The use of Yahoo! Kimo Knowledge+ as an example. Internet Research, 23(2), 133-159.

Lu, H., Chu, Y., Li, T., \& Wang, Q. (2013). Analysing the factors influencing learners' knowledge sharing behaviour in the virtual learning community of a faculty training programme. International Journal of Continuing Engineering Education and Life Long Learning, 23(3-4), 251-266.

Ma, M., \& Agarwal, R. (2007). Through a glass darkly: Information technology design, identity verification, and knowledge contribution in online communities. Information systems research, 18(1), 42-67.

Mairaru, S., Tyagi, S., Azeez, C., Sharma, D.C. (2019). Understanding the Print, Web, Television Media Habits and Preferences of Indians: A Uses and Gratification Perspective. Journal of Content, Community and Communication. 9(1), 75-80.

Majewski, G., Usoro, A., \& Khan, I. (2011). Knowledge sharing in immersive virtual communities of practice. Vine, 41(1), 41-62.

McMillan, D. W., \& Chavis, D. M. (1986). Sense of community: A definition and theory. Journal of community psychology, 14(1), 6-23.

McMillan, D. W. (1996). Sense of community. Journal of community psychology, 24(4), 315325.

Moher, D., Shamseer, L., Clarke, M., Ghersi, D., Liberati, A., Petticrew, M., ... \& Stewart, L. A. (2015). Preferred reporting items for systematic review and meta-analysis protocols (PRISMA-P) 2015 statement. Systematic reviews, 4(1), 1.

Nahapiet, J., \& Ghoshal, S. (1998). Social capital, intellectual capital, and the organizational advantage. Academy of Management Review, 23(2), 242-266.

Navimipour, N. J., \& Charband, Y. (2016). Knowledge sharing mechanisms and techniques in project teams: Literature review, classification, and current trends. Computers in Human Behavior, 62, 730-742. 
Nistor, N., Daxecker, I., Stanciu, D., \& Diekamp, O. (2015). Sense of community in academic communities of practice: Predictors and effects. Higher Education, 69(2), 257-273.

Oliver, R. L. (1980). A cognitive model of the antecedents and consequences of satisfaction decisions. Journal of marketing research, 460469.

Papadopoulos, T., Stamati, T., \& Nopparuch, P. (2013). Exploring the determinants of knowledge sharing via employee weblogs. International Journal of Information Management, 33(1), 133-146.

Park, J., \& Gabbard, J. L. (2018). Factors that affect scientists' knowledge sharing behavior in health and life sciences research communities: differences between explicit and implicit knowledge. Computers in Human Behavior, 78, 326-335.

Peters, C., Amato, C. H., \& Hollenbeck, C. R. (2007). An exploratory investigation of consumers' perceptions of wireless advertising. Journal of Advertising, 36(4), 129145.

Phang, C. W., Kankanhalli, A., \& Sabherwal, R. (2009). Usability and sociability in online communities: A comparative study of knowledge seeking and contribution. Journal of the Association for Information Systems, 10(10), 2.

Pirkkalainen, H., Pawlowski, J. M., Bick, M., \& Tannhäuser, A. C. (2018). Engaging in knowledge exchange: The instrumental psychological ownership in open innovation communities. International Journal of Information Management, 38(1), 277-287.

Shan, S., Xin, T., Wang, L., Li, Y., \& Li, L. (2013). Identifying influential factors of knowledge sharing in emergency events: a virtual community perspective. Systems Research and Behavioral Science, 30(3), 367382.

Shen, K. N., Yu, A. Y., \& Khalifa, M. (2010). Knowledge contribution in virtual communities: accounting for multiple dimensions of social presence through social identity. Behaviour \& Information Technology, 29(4), 337-348.

Shu, W., \& Chuang, Y. H. (2011). Why people share knowledge in virtual communities.
Social Behavior and Personality: an international journal, 39(5), 671-690.

Staples, D. S., \& Webster, J. (2008). Exploring the effects of trust, task interdependence and virtualness on knowledge sharing in teams. Information Systems Journal, 18(6), 617-640.

Tajfel, H. (1974). Social identity and intergroup behaviour. Information (International Social Science Council), 13(2), 65-93.

Tamjidyamcholo, A., Baba, M. S. B., Shuib, N. L. M., \& Rohani, V. A. (2014). Evaluation model for knowledge sharing in information security professional virtual community. Computers \& Security, 43, 19-34.

Tsai, H. T., \& Bagozzi, R. P. (2014). Contribution behavior in virtual communities: cognitive, emotional, and social influences. MIS Quarterly, 38(1).

Tseng, F. C., \& Kuo, F. Y. (2014). A study of social participation and knowledge sharing in the teachers' online professional community of practice. Computers \& Education, 72, 37-47.

Turner, J. C. (1982). Towards a cognitive redefinition of the social group. Social identity and intergroup relations, 15-40.

Usoro, A., Sharratt, M. W., Tsui, E., \& Shekhar, S. (2007). Trust as an antecedent to knowledge sharing in virtual communities of practice. Knowledge Management Research \& Practice, 5(3), 199-212.

Venkatesh, V., \& Goyal, S. (2010). Expectation disconfirmation and technology adoption: polynomial modeling and response surface analysis. MIS quarterly, 281-303.

Vroom, V. H. (1964). Work and motivation. 1964. NY: John Wiley \&sons, 45.

Wormald, R., \& Evans, J. (2018). What Makes Systematic Reviews Systematic and Why are They the Highest Level of Evidence?25(1), 2730.

Xiong, Y., Cheng, Z., Liang, E., \& Wu, Y. (2018). Accumulation mechanism of opinion leaders' social interaction ties in virtual communities: Empirical evidence from China. Computers in Human Behavior, 82, 81-93.

Xu, B., Li, D., \& Shao, B. (2012). Knowledge sharing in virtual communities: A study of citizenship behavior and its social-relational antecedents. International Journal of HumanComputer Interaction, 28(5), 347-359. 
Yan, Y., Davison, R. M., \& Mo, C. (2013). Employee creativity formation: The roles of knowledge seeking, knowledge contributing and flow experience in Web 2.0 virtual communities. Computers in Human Behavior, 29(5), 1923-1932.

Yan, Z., Wang, T., Chen, Y., \& Zhang, H. (2016). Knowledge sharing in online health communities: A social exchange theory perspective. Information \& Management, 53(5), 643-653.

Yao, C. Y., Tsai, C. C., \& Fang, Y. C. (2015). Understanding social capital, team learning, members'e-loyalty and knowledge sharing in virtual communities. Total Quality Management \& Business Excellence, 26(5-6), 619-631.

Ye, H. J., Feng, Y., \& Choi, B. C. (2015). Understanding knowledge contribution in online knowledge communities: A model of community support and forum leader support. Electronic Commerce Research and Applications, 14(1), 34-45.

Yen, C. (2016). How to unite the power of the masses? Exploring collective stickiness intention in social network sites from the perspective of knowledge sharing. Behaviour \& Information Technology, 35(2), 118-133.

Yoon, C., \& Rolland, E. (2012). Knowledgesharing in virtual communities: familiarity, anonymity and self-determination theory. Behaviour \& Information Technology, 31(11), 1133-1143.

Yuan, D., Lin, Z.,\& Zhuo, R. (2016). What drives consumer knowledge sharing in online travel communities?: Personal attributes or e- service factors?. Computers in human behavior, 63, 68-74.

Yu, T. K., Lu, L. C., \& Liu, T. F. (2010). Exploring factors that influence knowledge sharing behavior via weblogs. Computers in human behavior, 26(1), 32-41.

Zboralski, K. (2009). Antecedents of knowledge sharing in communities of practice. Journal of Knowledge Management, 13(3), 90101.

Zhang, Y., Fang, Y., Wei, K. K., \& Chen, H. (2010). Exploring the role of psychological safety in promoting the intention to continue sharing knowledge in virtual communities. International Journal of Information Management, 30(5), 425-436.

Zhang, X., Liu, S., Deng, Z., \& Chen, X. (2017a). Knowledge sharing motivations in online health communities: A comparative study of health professionals and normal users. Computers in Human Behavior, 75, 797810.

Zhang, D., Zhang, F., Lin, M., \& Du, H. S. (2017b). Knowledge sharing among innovative customers in a virtual innovation community: the roles of psychological capital, material reward and reciprocal relationship. Online Information Review, 41(5), 691-709.

Zhao, J., Abrahamson, K., Anderson, J. G., Ha, S., \& Widdows, R. (2013). Trust, empathy, social identity, and contribution of knowledge within patient online communities. Behaviour \& Information Technology, 32(10), 1041-1048. 